\title{
Experimental approach about fracture process in timber elements under mechano-sorptive effects
}

\author{
F. Dubois ${ }^{\mathrm{a}}$, O. Pop, J.-M. Husson, and N. Sauvat \\ University of Limoges, Heterogeneous Material Research Group, Civil Engineering and Durability \\ Department, 19300 Egletons, France
}

\begin{abstract}
This paper deals with the fracture problem in timber structures subject to mechanical and climatic loadings. Mechanical tests illustrate local and global fracture properties at different moisture content levels on DCB specimens of douglas fire. It is shown the dependence of moisture content in terms of compliance and energy release rate. To highlight the hygro-mechanical coupling, tests employ an electromechanical press associated with an environmental chamber in which humidity and air temperature are regulated. Two families of tests are performed, the first in dry state conditions and the second in a wet state conditions. The main objective of this paper is to fix first experimental results in order to integrate, in future works, mechano-sorptive effects on the crack growth process by taking into account moisture content variations in the crack tip vicinity associated with sorption kinetics.
\end{abstract}

\section{Introduction}

Timber elements placed in outdoor conditions are solicited by mechanical loadings and climatic variations. The timber behaviour is usually interpreted as a mechano-sorptive coupling mechanical fields and moisture content gradients. In the past, a lot of works has been developed in order to understand this interaction at a material scale and for bending behaviour. If we take the example of timber joints, the toughness can be accelerate by moisture content variations by provoking a crack initiation and the crack propagation until a partial collapse [1]. In this context, in fracture mechanics, the one difficulty meet by the scientist community is to put in evidence the mechano-sorptive effects in the fracture mechanic kinetic. Several scientific explorations have shown that coupling between moisture content variations and mechanical loading is subject to shrinkage-swelling effects and a modification of elastic or viscoelastic properties. In terms of thermodynamic visions, the last phenomenon is traduced, for constant mechanical loadings, by a increase of compliance properties during moistening phases and a blocking of strains during drying process corresponding to a partial storage of the free energy [2]. Then, these last observations requests to put in evidence the local fracture behaviour in moistening and drying phases. However, before highlighting the mechanosorptive effects during wetting and drying phases of, it is necessary to characterize the mechanical properties for dry and wet conditions.

a e-mail : frederic.dubois@unilim.fr

This is an Open Access article distributed under the terms of the Creative Commons Attribution-Noncommercial License 3.0, which permits unrestricted use, distribution, and reproduction in any noncommercial medium, provided the original work is properly cited. 
The first section deals with the experimental setup based on a double cantilever beam specimen in douglas fire using a electromechanical testing machine associated with a regulated environment chamber. These tests allow the determination of the specimen compliance and the critical energy release rate versus the crack tip position.

Dry and wet fracture behaviour characterisation is proposed in the second session. This part allows the definition fracture parameters versus moisture content level as global stiffness, energy release rate, etc.

A last part presents an original approach for taking into account mechano-sorptive effects in fracture mechanics. This model relies primarily by blocking the effects of deformation during drying phases.

\section{Mechanical tests under dry and wet conditions}

\subsection{Experimental setup}

The experimental tests are based on the use of a double cantilever beam in Douglas fire submitted at a force control loading. Two sample groups are conditioned in dry and wet environments, respectively. In the other hand, for initial moistened specimens, a ramp of drying is imposed. All tests are filmed by CCD camera in order to record the crack tip advance. The douglas fire specimen is a Double Cantilever Beam, Fig 1. The initial crack is oriented in the longitudinal axis with an initial length of $50 \mathrm{~mm}$. Its thickness is $20 \mathrm{~mm}$. The loading axes have a diameter of $10 \mathrm{~mm}$. The crack propagation is assumed to be in the Radial Longitudinal plane.
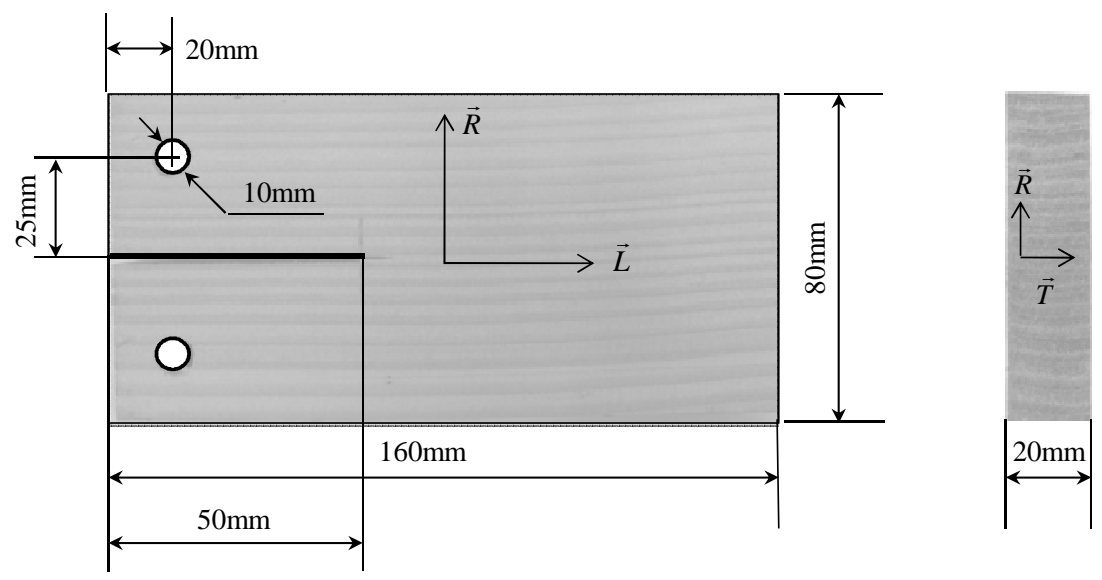

Fig. 1. DCB specimen

Two families of four specimens are placed in a dry $\left(20^{\circ} \mathrm{C}, 40 \% \mathrm{HR}\right)$ and wet $\left(20^{\circ} \mathrm{C}, 85 \% \mathrm{HR}\right)$ climate corresponding to moisture contents of approximately $6 \%$ and $22 \%$, respectively. These specimens are acclimated during several weeks in order to obtain a moisture content homogeneity. The fracture mechano-sorptive behaviour is stud by employing an electro mechanic machine incorporating an environmental chamber allowing the time synchronization between forcedisplacement and temperature/humidity histories, Fig. 2. The experimental device is completed by monitoring the crack tip advance by a CCD camera. The synchronization between images and mechanical data is permitted by using a tracking marker technique of the point of force application. During tests, the environment chamber is regulated in order to impose the equivalent moisture content conditioning. In order to limit creep effects, we impose a high constant displacement speed of $0,5 \mathrm{~mm} / \mathrm{min}$ until the total specimen collapse. In these conditions, the total test time does not exceed 4 minutes. 

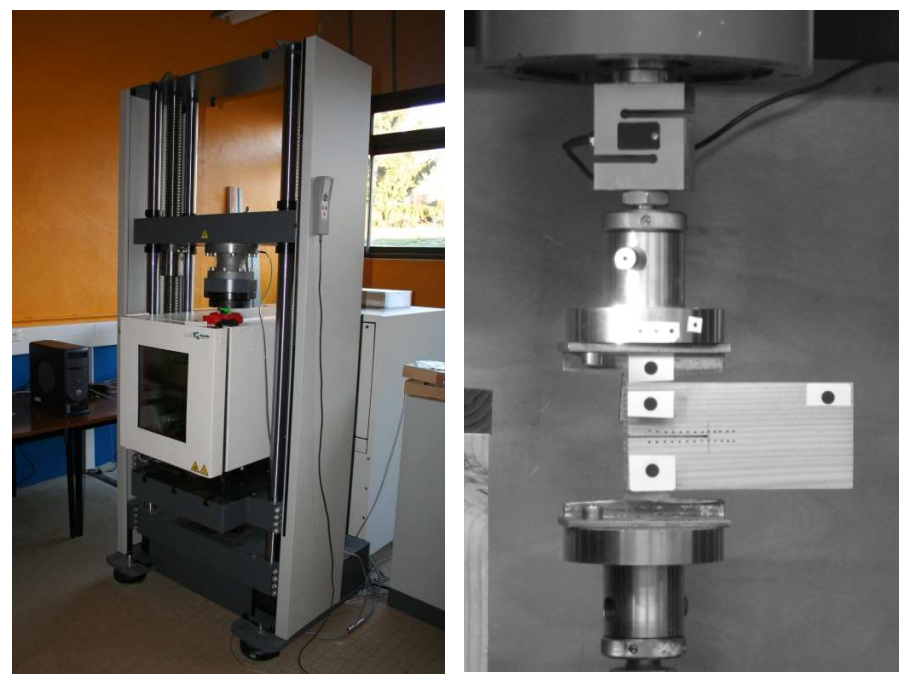

Fig. 2. Testing machine and its environmental chamber

\section{2 fracture parameter characterisation}

Mechano-sorptive effects being a coupling between moisture content variations and time dependent behaviour (viscoelasticity), fracture mechanic must be approached considering energy concepts. According to force-displacement curves, we propose an energetic balance approach in order to characterize fracture parameters in terms of compliance evolution and energy release rate. Let us consider an elastic behaviour in which the crack growth kinematic can be separate into two steps. According to the cohesive theory, a process zone is developed in the crack tip vicinity which can be assimilated to a damage volume dissipating an energy named $W_{D}$. The second step corresponds on the crack tip advance in this process zone by crack lip debonding inducing an additional dissipation noted $W_{s}$, Fig. 3. If $W_{\text {ext }}$ is the external force-displacement work, the energy balance during the crack process can be written as follow, [3], [4]:

$$
W_{e x t}=U_{e}+W_{D}+W_{s}
$$

in which $U_{e}$ designates the free energy temporarily stored in the material.

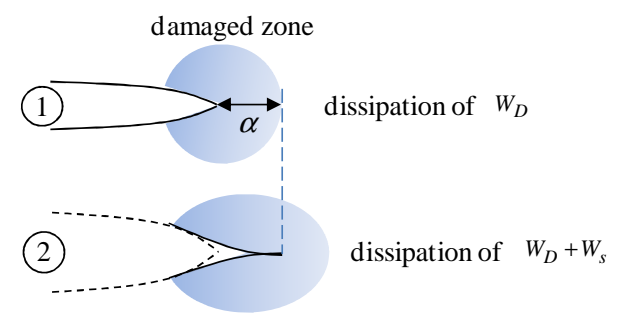

Crack propagation in the process zone

Fig. 3. Energy repartition during crack propagation

The separation between the two dissipation sources $\left(W_{D}\right.$ and $\left.W_{s}\right)$ is an actual scientific problem. Several authors treat this problematic by considering a virtual crack length in order to confuse 
damage and crack tip evolutions. Fig. 4 illustrates different mechanical parameters in the forcedisplacement graph [5].

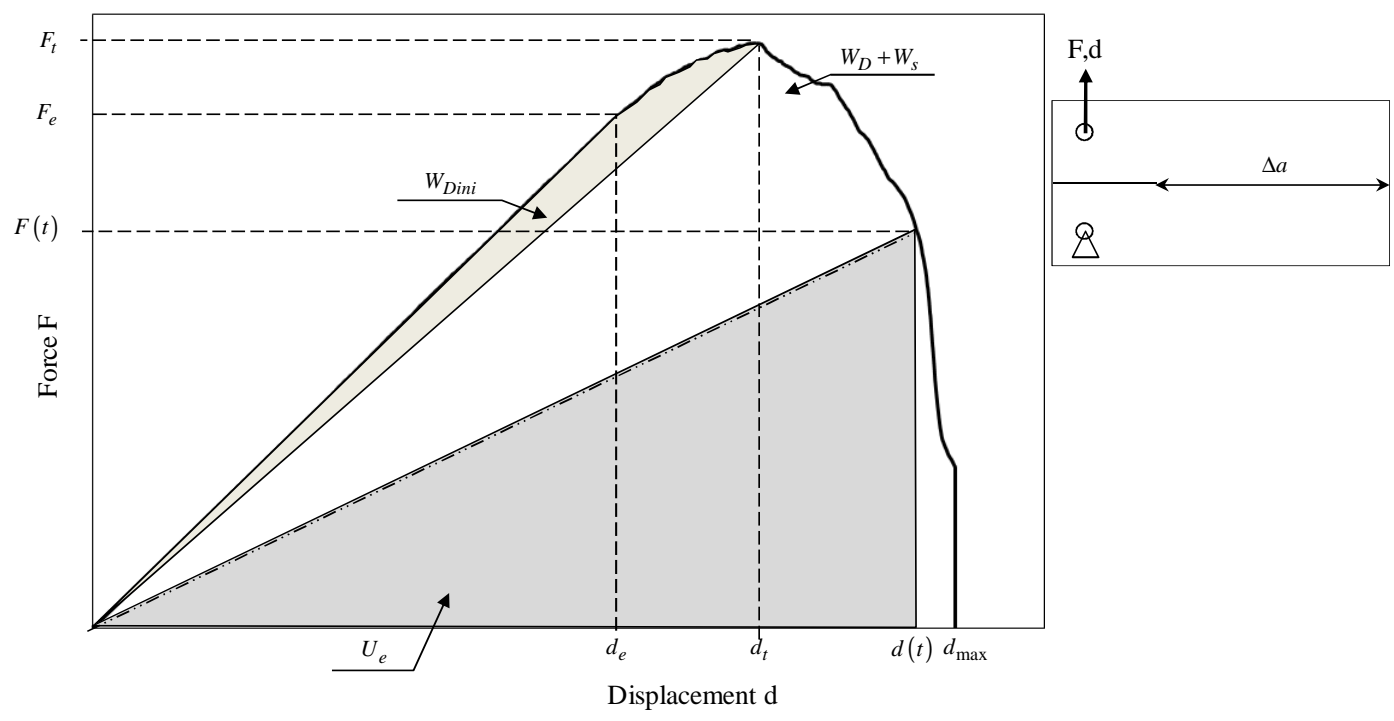

Fig. 4. Properties of the force-displacement curve

The unstable crack growth of the DCB specimen allows the definition of following properties:

- $F_{e}, d_{e}$ are the force and displacement corresponding to the damage initiation

- $F_{t}, d_{t}$ are the force and displacement corresponding to the crack growth initiation.

- $W_{\text {Dini }}$ is the damage dissipation before the crack growth initiation

- $d_{\text {max }}$ designates the max displacement corresponding to the final specimen collapse

This graphic allows the evaluation of the instantaneous specimen stiffness $K_{i n i}$ such as:

$$
K_{\text {ini }}=\frac{F_{e}}{d_{e}}
$$

For a given experimental time $t$, the external work is given by:

$$
W_{\text {ext }}=\int_{0}^{t} F(\tau) \cdot \frac{\partial d}{\partial \tau} d \tau
$$

The free energy $U_{e}$ is graphically defined by supposing a neglected plasticity as follow:

$$
U_{e}(t)=\frac{1}{2} \cdot F(t) \cdot d(t)
$$

Finally, the crack separation work $G_{c}$ is evaluated as the ratio of the total dissipation energy by the full crack path $\Delta a$ :

$$
G c=\frac{W_{D}\left(d_{\max }\right)+W_{s}\left(d_{\max }\right)}{\Delta a}
$$

\subsection{Experimental results}

Experimental tests are performed for the dry and wet conditions. Fig. 5 shows a classical result in which equations (1), (3) and (4) allow the plotting of the balance energy versus experimental 
displacements. Fracture parameters are indicated in tables 1 and 2 for dry and wet conditions, respectively.

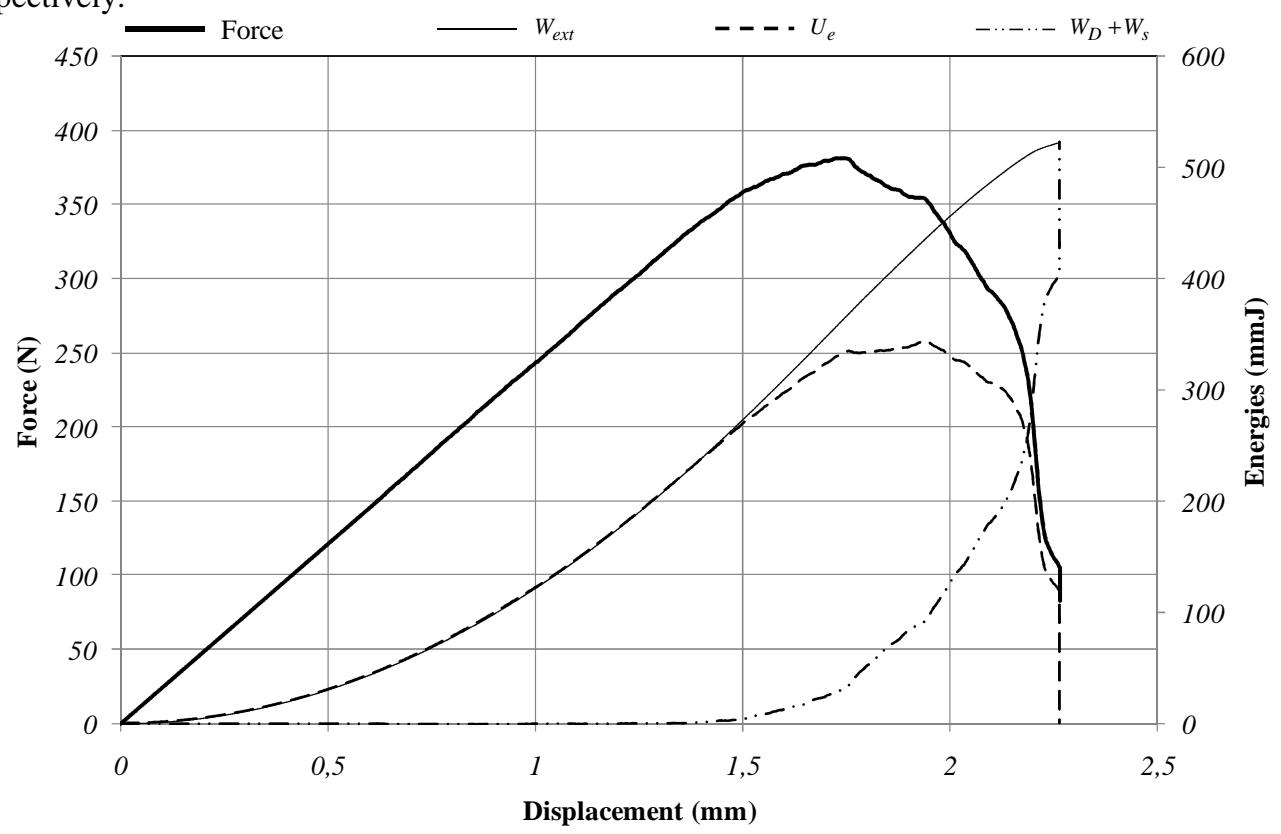

Fig. 5. Experimental result type

Table 1. Fracture parameters for dry samples $(w=6 \%)$

\begin{tabular}{|c|c|c|c|c|c|c|c|c|}
\hline Sample & $\rho_{o}$ & $\begin{array}{c}K_{\text {ini }} \\
(\mathrm{N} / \mathrm{mm})\end{array}$ & $F_{e}(\mathrm{~N})$ & $\begin{array}{c}d_{e} \\
(\mathrm{~mm})\end{array}$ & $F_{t}(\mathrm{~N})$ & $\begin{array}{c}d_{t} \\
(\mathrm{~mm})\end{array}$ & $V_{\text {Dini }}(\mathrm{mJ})$ & $\begin{array}{c}G_{c} \\
\left(\mathrm{~J} / \mathrm{m}^{2}\right)\end{array}$ \\
\hline 1 & 0,47 & 496 & 285 & 0,58 & 383 & 1,21 & 81 & 184 \\
\hline 2 & 0,43 & 370 & 330 & 0,90 & 372 & 1,27 & 46 & 196 \\
\hline 3 & 0,47 & 693 & 284 & 0,42 & 358 & 0,64 & 17 & 144 \\
\hline $\begin{array}{c}\text { average } \\
\text { values }\end{array}$ & 0,46 & 666 & 451 & 0,71 & 521 & 1,05 & 59 & 208 \\
\hline
\end{tabular}

Table 2. Fracture parameters for wet samples ( $w=22 \%$ )

\begin{tabular}{|c|c|c|c|c|c|c|c|c|}
\hline Sample & $\rho_{o}$ & $\begin{array}{c}K_{\text {ini }} \\
(\mathrm{N} / \mathrm{mm})\end{array}$ & $F_{e}(\mathrm{~N})$ & $\begin{array}{c}d_{e} \\
(\mathrm{~mm})\end{array}$ & $F_{t}(\mathrm{~N})$ & $\begin{array}{c}d_{t} \\
(\mathrm{~mm})\end{array}$ & $V_{\text {Dini }}(\mathrm{mJ})$ & $\begin{array}{c}G_{c} \\
\left(\mathrm{~J} / \mathrm{m}^{2}\right)\end{array}$ \\
\hline 1 & 0,53 & 242 & 251 & 1,00 & 379 & 1,76 & 37 & 238 \\
\hline 2 & 0,56 & 440 & 350 & 0,80 & 464 & 1,22 & 37 & 223 \\
\hline 3 & 0,54 & 485 & 605 & 1,25 & 605 & 1,25 & 0 & 304 \\
\hline $\begin{array}{c}\text { average } \\
\text { values }\end{array}$ & 0,52 & 533 & 338 & 0,64 & 511 & 1,26 & 89 & 210 \\
\hline
\end{tabular}

By comparing dry and wet results, we can do few remarks. Firstly, the global stiffness decreases for high moisture content levels. However, the max effort and the crack separation work rate increase with moisture content. These conclusions can be illustrated in Fig. 6 in which it is compared average force evolution for dry and wet tests. 


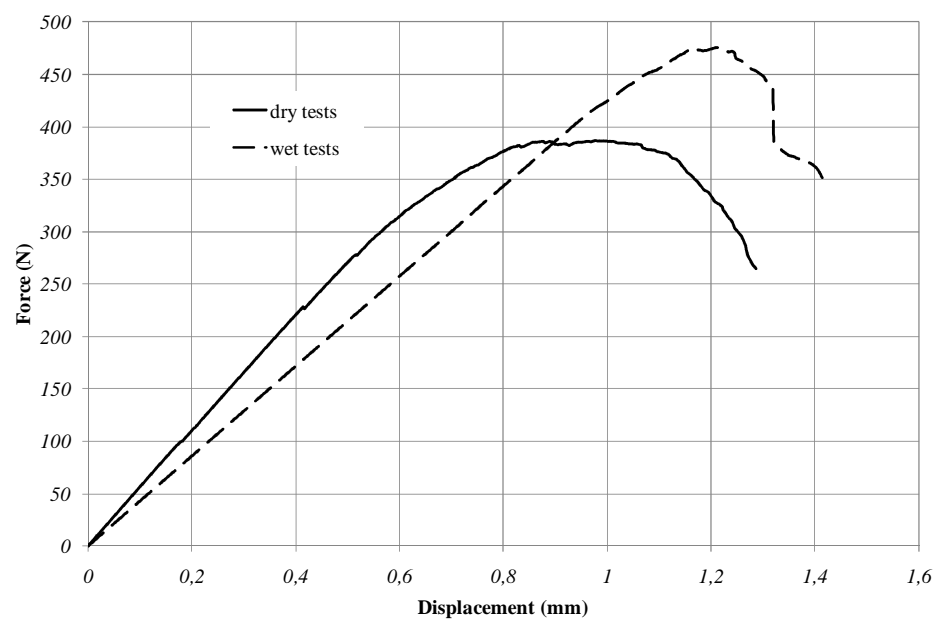

Fig. 6. Average results for force-displacement curves in dry and wet conditions

\section{Mechanical tests under variable climate}

The mechanical test under variable climate conditions is based on a creep loading accompanied by relative humidity changes. This test allows the introduction, in the fracture behaviour, effects of the viscoelasticity, the shrinkage or swelling and mechano-sorptive process. Let us limit our investigation to drying process.

\subsection{Loading history}

The association of the mechanical loading with the decrease of the relative humidity requests to introduce the time as synchronisation variable. In your case, we choose to load the specimen conditioned in a wet environment $\left(20^{\circ} \mathrm{C}, 85 \% \mathrm{HR}\right)$.

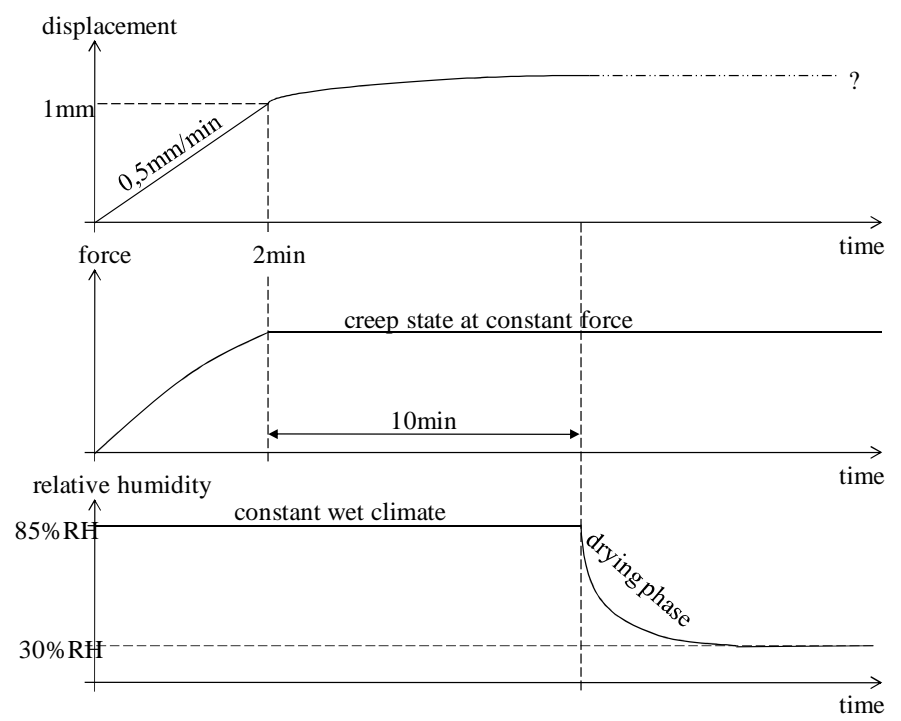

Fig. 7. Hygro-mechanic loading history 


\section{4th International Conference on Experimental Mechanics}

According to results shown in table 2, the loading value is chosen according to a static displacement of $1 \mathrm{~mm}$ with a displacement speed of $0,5 \mathrm{~mm} / \mathrm{min}$. At this time, the force is fixed as constant during 10 minutes in order to observe creep response in constant wet climate. The next step is characterised by a drying phase by changing climate conditions at a dry state $\left(20^{\circ} \mathrm{C}, 30 \% \mathrm{HR}\right)$. The experimenters expect now the complete collapse of the sample. Fig. 7 summarizes the hygromechanical loading.

\subsection{Experimental results}

The force-displacement curve is posted in Fig. 8. The graph can be separated in four specific zones.

1- This zone corresponds to the loading of the wet sample with a displacement speed of $0,5 \mathrm{~mm} / \mathrm{min}$. The overall stiffness is equal to $260 \mathrm{~N} / \mathrm{mm}$. According to a finale displacement of $1 \mathrm{~mm}$, the corresponding force is $355 \mathrm{~N}$.

2- During ten minutes, the force is kept constant. The sample is in a creep configuration. We can observe the displacement evolution versus time. At this state, the relative humidity is maintained at $85 \%$.

3- At this time, the phase of drying starts. According to diffusion process, the specimen begins to dry from the outside. The creep response results on the combination of mechano-sorptive and shrinkage effects. We can observe a displacement blocking characterising of mechano-sorptive effect and its hygro-lock properties [6], [7].

4- The last phenomenon is characterised by a continuum increase of displacements. According to a non linear behaviour, this phase can be assimilated at a secondary creep state accentuated by the $3 \mathrm{D}$ diffusion process. The total collapse can be observed after a total time of $2 \mathrm{~h} 40$.

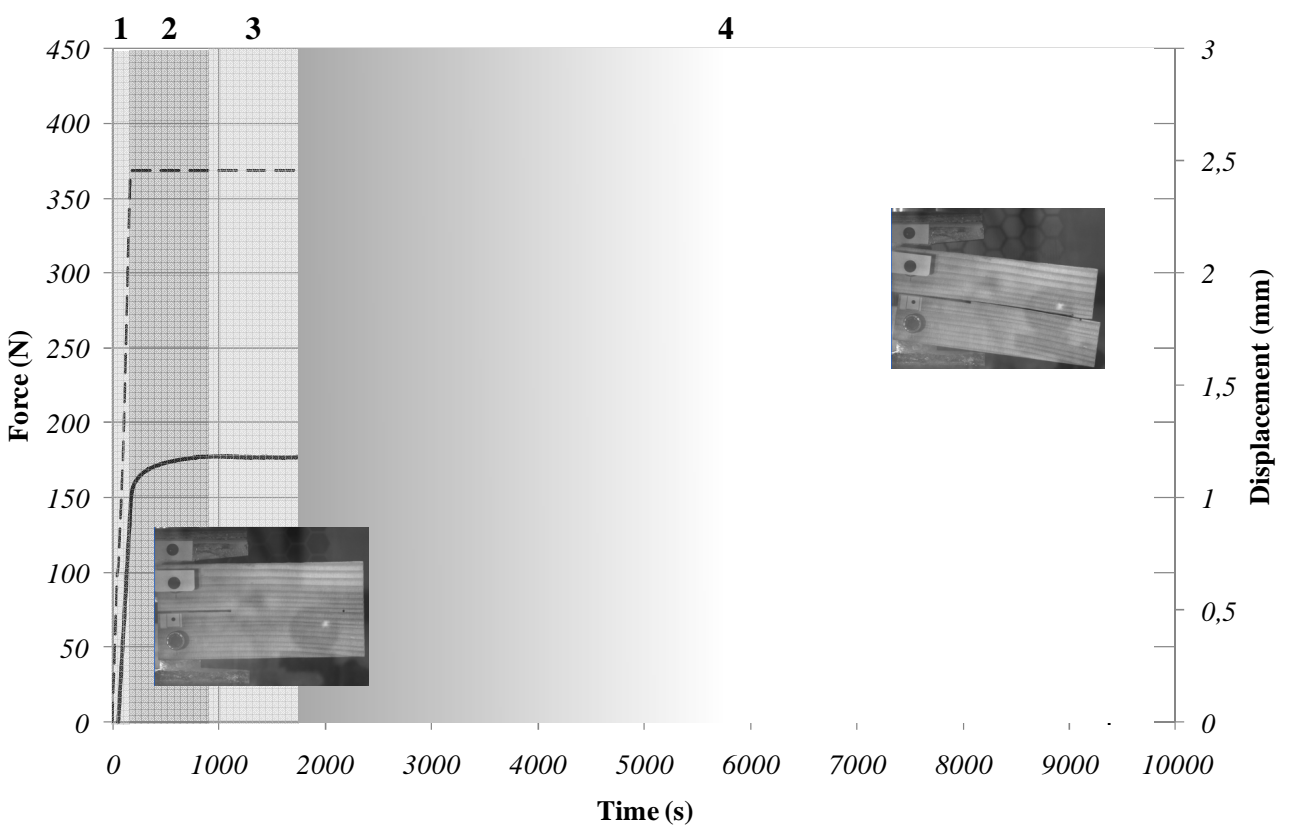

Fig. 8. Experimental results 


\section{Conclusions and perspectives}

This work presents an experimental protocol to demonstrate the fracture behaviour of a DCB specimen submitted a mechanical loading coupled with climatic variations. In this context, a first experimental phase allows to capture the fracture behaviour in dry and wet environments. The second experimental phase presents an original approach of cracking. It helps to highlight the effects of climate change on the boot of the crack. We are limited to periods of drying appearing to be most binding. Observed results demonstrate the acceleration of the initiation and propagation of crack during the drying phase. The experimental observations, in terms of evolution of displacement versus time, put in evidence similar effects encountered in mechano-sorptive responses. This work must be completed by similar tests under moistening phases. In order to understand this complex behaviour, it seems necessary to introduce mechano-sorptive approach into the fracture behaviour in order to illustrate it thru physical interpretations and thermodynamical considerations.

\section{References}

1. M. Chaplain, T. Dethan, G. Valentin, 12th ICF12 (2009)

2. J.M. Husson, F. Dubois, N. Sauvat, Mechanics of Time-Dependent Materials (2009)

3. F. Dubois, C. Chazal, C. Petit, Int. J. of Fract. 113, 21 (2002)

4. F. Dubois, C. Petit, Engineering Fracture Mechanics, 72, 15, 2005

5. S. Morel, Int. J. of Sol. and Struct. (2007

6. M. Husson, F. Dubois, N. Sauvat, Eur. J. of Env. and Civ. Eng. 12/9, 12 (2008)

7. Gril, J., International COST 508 Wood mechanics Conference, (1996) 